\title{
Errores de diagnóstico en la práctica médica
} Diagnostic errors in medical practice

El cambio de cultura acerca del error en medicina es uno de los desafíos más trascendentes de la medicina y de otras disciplinas que participan en el cuidado de la salud. Este cambio, que comenzó hace más de 20 años, aún se ha extendido muy escasamente por lo cual tenemos un desafío enorme por delante. No obstante, y aunque lentamente, varios logros se alcanzaron en la seguridad del paciente que contribuyen a reducir los errores y disminuir los riesgos en la atención médica.

Los errores de diagnóstico han merecido recientemente más atención y se está comenzando a conocer mejor sus mecanismos y eventos adversos. Se desconoce su verdadera frecuencia aunque nuestras decisiones incorrectas son más frecuentes de lo que creemos ya que se estima que el error no es inferior a $25 \%$ de los diagnósticos. Una revisión sistemática evaluó miles de autopsias desde 1966 a 2002, detectando errores de diagnóstico como posible causa de muerte. La tasa media de errores fue de $23,5 \%$ (Shojania KG et al. JAMA 2003).

Es de destacar que los errores de diagnóstico ocasionan más eventos adversos que otros, que alcanzan un $15 \%$ a $20 \%$ y son los más difíciles de evitar. A pesar de estos datos muy preocupantes, la literatura médica es más escasa que en otros errores.

Asimismo, es de notar que representan la principal causa de juicios por mala praxis y superan ampliamente a las demandas en cirugía y los errores de medicación.

Hay varios factores desfavorables en la práctica médica que influyen en las fallas de diagnóstico, en especial al interferir perniciosamente en la relación médico-paciente. La realidad creciente de consultas cada vez más breves motivan un muy escaso diálogo con el paciente o los padres y por lo tanto no se los escucha. Desde tiempos ancestrales nuestro lenguaje fue instrumento de indagación y de conocimiento sustentado en tres condiciones imprescindibles: empatía, habilidades de comunicación y el tiempo necesario para que la palabra desplegara toda su potencia. Hoy en día, esto es un bien infrecuente ya que por varios motivos la comunicación se desplazó desde el diálogo hacia una larga serie de sustitutos tecnológicos.

Asimismo, actualmente hay un enorme acceso a la información médica, la mayoría irrelevante y sumamente mayor que la necesaria para ser un buen médico. Esto puede llevar al riesgo, tal vez ya hemos llegado, de que aquellos médicos que sólo tengan copiosa y nueva información supongan que también saben mucho, lo cual, suele ser justo lo contrario.

\section{¿Cuáles mecanismos intervienen en el error de diagnóstico?}

Una de las causas principales son las fallas o sesgos en la cognición, aunque hay que destacar que no es mayormente la falta de conocimiento lo que genera errores, sino los problemas en el pensamiento del médico.

El pensamiento es una acción sujeta a reglas, plagada de cortocircuitos automáticos, de estereotipos y del que raramente tomamos conciencia. No es habitual que los médicos expliciten como piensan; la pausa reflexiva imprescindible para sacar a la luz las formas de su razonamiento, formular hipótesis, extraer conclusiones, suelen no estar en nuestra agenda cotidiana.

Una falla cognitiva frecuente en el pensamiento, no saber que uno no sabe, provoca que el médico crea que el diagnóstico que realizó es apropiado o que tomó la decisión correcta, cuando no es así. En gran medida, esto ocurre porque hay ausencia del deseo y capacidad de reflexión sobre el proceso del pensamiento y asimismo, examinar críticamente nuestro juicio clínico antes tomar las decisiones. Es de señalar, que los diagnósticos erróneos mayormente no ocurren en enfermedades graves sino en las más comunes.

Varios mecanismos están involucrados en las fallas cognitivas, principalmente en los complejos mediante los cuales nuestros cerebros reciben y procesan la información. Los psicólogos que estudian la cognición han aportado grandes contribuciones, tales como la vulnerabilidad de la mente a los sesgos cognitivos, las falacias en el pensamiento lógico, las falsas presunciones y otras fallas del razonamiento. Está comprobado que nuestro pensamiento es defectuoso en varias acciones que realizamos cotidianamente.

Los dos modos principales en este mecanismo se denominan automático y controlado o "intuitivo" y "analítico" en el lenguaje habitual. El proceso intuitivo es innato, evoluciona a través de la experiencia y requiere escaso razonamiento porque mayormente es reflejo y automático. Asimismo, es subconsciente y rápido lo cual nos permite manejar gran parte de nuestras acciones 
diarias en todos los campos de la actividad humana. En general, avanzamos en la vida yendo de una de las asociaciones del modo intuitivo a la siguiente en una sucesión de patrones de acción en su mayor parte mecánicos. Aun cuando resultan indispensables, en ellos están presentes las fallas cognitivas y la mayoría de los sesgos y fracasos del pensamiento.

La intuición es una muy buena cualidad y suele dar adecuados resultados en la asistencia clínica, podemos confiar en ella pero en todo momento debemos tener presente que inevitablemente fallará con algunos pacientes ya que no siempre es lo suficientemente fiable. Aquí, la actitud crítica y la auto reflexión nos ayudarán, ya que son aspectos esenciales para alcanzar la práctica de una buena medicina.

Los procesos analíticos, en cambio, son conscientes, deliberados, más lentos y generalmente confiables. Siguen las leyes de la ciencia y la lógica, por lo cual es probable que sean racionales. No obstante, pueden ocurrir fallas, pero en general suceden cuando se siguen reglas equivocadas o intervienen otros factores, escaso tiempo en la consulta, exceso de trabajo, cansancio, falta de sueño, trastornos emocionales, estrés, etc. El mayor inconveniente del razonamiento analítico es que necesita muchos recursos y en la mayoría de los campos de la medicina sería poco práctico e improbable resolver cada decisión en forma analítica.

Otro aspecto que contribuye al error en este campo es el exceso de confianza del médico en la precisión de sus diagnósticos, algo que se produce cuando la relación entre precisión en el juicio clínico y la confianza no están bien calibradas debido a que la confianza es mayor de lo que debiera ser.

\section{¿A qué se puede deber esta actitud?}

Muchos profesionales tienen el concepto de que "saben todo lo que deben saber", una actitud omnipotente y arrogante que expresa un desinterés hacia cualquier modificación de la conducta. En esta conducta juega un rol trascendente la falla cognitiva que ya señalamos: no saber lo que no sabe. La frase de Charles Darwin "La confianza es más un producto de la ignorancia que del conocimiento", es sin duda aplicable a esta situación.

La actitud dominante del exceso de confianza puede enmascarar las dudas en la práctica médica, esto suele llevar a no tolerar la incertidumbre y suponer que en la medicina prevalece la certeza. La tendencia a un exceso de confianza en los diagnósticos podría relacionarse a que si bien los médicos tienen conciencia de la posibilidad del error, suelen creer que, quienes los cometen, son otros.

Asimismo, una revisión de la práctica médica en Estados Unidos encontró que una alta proporción de médicos no sigue las guías de las sociedades médicas. Por ejemplo, en la evaluación del tratamiento de la hipercolesterolemia se observó que el 95\% de los médicos conocía las recomendaciones pero sólo el 18\% las seguía.

En un estudio reciente (Meyer AN et al. JAMA Intern Med 2013) se halló, entre otras cosas, que la precisión diagnóstica disminuía cuando los médicos enfrentaban casos más difíciles. Sin embargo, en estas situaciones la confianza casi no se modificó, algo que es opuesto a lo esperado ya que tanto la precisión como la confianza deberían disminuir frente a esos casos. Los autores concluyen que no habría buena asociación entre la precisión diagnóstica de los médicos y su confianza en esa precisión, y que mejorar estas asociaciones podría disminuir el error diagnóstico.

\section{¿Qué podemos hacer?}

Como ya señalamos, es cierto que los problemas que subyacen en los sistemas de atención de la salud contribuyen a diagnósticos equivocados y demorados, por lo cual es perentorio que mejoren. Pero también es cierto que los sesgos cognitivos de los médicos juegan un papel trascendente en gran parte de los errores de diagnóstico y por lo tanto, el lograr mejorar, está en cada uno de nosotros. Para eso es imprescindible que la psicología cognitiva esté considerada dentro de las competencias de la medicina a fin de que podamos lograr el objetivo principal de involucrar a estudiantes y médicos en la "metacognición" (reflexionar sobre su propio pensamiento), con la esperanza de que van a conocer algo de su mal uso de la heurística (técnica de la indagación) antes de que causen daño.

Las continuas fallas en los errores del pensamiento en la toma de decisiones médicas, pone en grave riesgo la seguridad del paciente. Muchos médicos no conocen sus limitaciones o no les interesan los aspectos sobre sus decisiones; en especial debido a que no han cultivado desde la universidad el pensamiento crítico que los lleve a la reflexión profunda de sus acciones.

Es por lo tanto que estas recomendaciones no se deben considerar como opcionales, sino como una parte integral de la educación médica, un principio ético ineludible, y por extensión, un deber moral y profesional.

\section{Dr. José M. Ceriani Cernadas} Editor

http:/ /dx.doi.org/10.5546/aap.2015.194 